\title{
Densidad hepática promedio y su asociación con el desarrollo de síndrome metabólico
}

\section{Average of liver density and its association with development of}

\section{metabolic syndrome}

Fernando Ramírez-Trejo ${ }^{1 *}$, Javier J. Onofre-Castillo", Ricardo D. Martínez-López¹,

Ricardo Gutiérrez-Castaño ${ }^{1}$, Enrique Torres-Gómez ${ }^{1}$, Ana Córdova-Butler ${ }^{1}$ y Abraham Álvarez-Rosales ${ }^{2}$

${ }^{1}$ Departamento de Imagenología Diagnóstica y Terapéutica, Hospital Christus Muguerza; ${ }^{2}$ Departamento de Cirugía Ginecológica de Mínima Invasión,

Universidad de Monterrey UDEM, San Pedro Garza García. Monterrey, Nuevo León, México

\section{RESUMEN}

Objetivo: Correlacionar los valores de unidades Hounsfield (UH) reportados en el hígado con las alteraciones bioquímicas en pacientes asintomáticos. Método: Estudio longitudinal y retrospectivo en pacientes a quienes se realizó tomografía de abdomen y estudios de laboratorios de química sanguínea de enero de 2018 a agosto de 2019. Se utilizó un tomógrafo General Electric Revolution (GE Milwaukee) de 256 detectores y una estación de reformateo en el sistema RIS-PACS Carestream. Se colocaron regiones de interés circulares de $1 \mathrm{~cm}$ de diámetro y se realizó un promedio total que se integró a la base de datos. La medición del perímetro abdominal se obtuvo a nivel de L3 en un corte axial estricto. Resultados: Se analizaron 63 pacientes y se encontró una correlación de Pearson estadísticamente significativa $(\mathrm{p}<0.005)$. Al analizar las coordenadas en curva ROC de cruce de las UH y la presencia o no de síndrome metabólico se encontró que el valor de 45.4 tiene la mayor utilidad, con una sensibilidad del 82\% y una especificidad del $72 \%$. Conclusiones: El valor obtenido de $45.4 \mathrm{UH}$ puede ayudarnos en una tomografía simple para poder identificar como corte inferior a pacientes con alta probabilidad de no presentar alteraciones metabólicas, y reducir los falsos positivos para reportes de esteatosis.

Palabras clave: Enfermedad de hígado graso no alcohólico. Esteatosis hepática. Tomografía. Síndrome metabólico. 


\section{ABSTRACT}

Objective: To correlate the values of Hounsfield units reported in the liver with biochemical alterations in asymptomatic patients. Method: Longitudinal and retrospective study in patients who attended imaging studies and an abdominal tomography and blood chemistry laboratory studies were performed from January 2018 to August 2019. A 256 detector Revolution CT scanner was used (GE Milwaukee) and measurements were taken the reformatting station in Carestream RIS-PACS placing $1 \mathrm{~cm}$ in diameter ROIs in liver segments obtaining a total average of the liver density. The abdominal circumference was obtained at the body of L3 vertebrae in axial cuts, both measurements were integrated in the database. Results: 63 patients were analyzed, finding a statistically significant Pearson's correlation ( $p<0.005)$. Analyzing the curved coordinates of the ROC of the UH and the presence or absence of metabolic syndrome, it was found that the value of 45.4 has greater utility with a sensitivity of $82 \%$ and a specificity of $72 \%$. Conclusions: The value obtained from 45.4 Hounsfield units, we can use a simple tomography to identify patients with a high probability of not having metabolic alterations as a lower cut, and to reduce false positives for reports of steatosis.

Key words: Non-alcoholic fatty liver disease (NAFLD). Steatosis. Tomography. Metabolic syndrome.

\section{INTRODUCCIÓN}

Con el importante aumento de la obesidad en todo el mundo, y en mayor número en México, la morbilidad y la mortalidad que se asocian también han aumentado. Uno de los hallazgos más habituales en estos pacientes es la enfermedad de hígado graso, la cual se ha vuelto una epidemia. La enfermedad de hígado graso no alcohólico (EHGNA) tiene un espectro importante de gravedad, desde esteatosis hasta esteatohepatitis con progresión a fibrosis, cirrosis y carcinoma hepatocelular ${ }^{1}$.

La esteatosis hepática se caracteriza por la acumulación de triglicéridos en macrovesículas y microvesículas en más del 5\% de los hepatocitos, distribuyéndose principalmente en la región perivenular; las áreas periportales suelen encontrarse respetadas. A pesar de que la fisiopatología no se conoce exactamente, entre las causas más importantes se ha encontrado la resistencia a la insulina ${ }^{2}$, debido a que se produce una falla en la supresión del flujo de los ácidos grasos, causando una acumulación de estos en el hígado.

En la actualidad es la enfermedad hepática crónica más común en el mundo, y se estima que en México el promedio del sobrepeso es de un $38 \%$ y que un $21 \%$ de la población es obesa. Un estudio realizado en México reporta una prevalencia de esteatosis en pacientes obesos del 17\%, y de estos, el $82 \%$ presentan síndrome metabólico ${ }^{3}$.

La evaluación por tomografía de la EHGNA se basa en los valores de atenuación del parénquima hepático en unidades Hounsfield (UH). El valor de atenuación de la grasa (aproximadamente -100 UH) es mucho menor que el de los tejidos blandos, y por ende 
disminuye el promedio total de los valores del parénquima ${ }^{4}$. En radiología se toma en cuenta el valor estandarizado de atenuación hepática de aproximadamente 55-65 UH, o no más de 15 UH de diferencia con el bazo $(\mathrm{CT}-\mathrm{ls})^{5,6}$. Se ha documentado que aproximadamente 1.6 UH de disminución corresponden a $1 \mathrm{mg}$ de triglicéridos alojados en el parénquima ${ }^{7}$.

Es ampliamente aceptado que los valores del perfil lipídico y las transaminasas no son lo suficientemente específicos ni lo bastante sensibles como para detectar EHGNA; sin embargo, se ha observado que existen cifras elevadas de colesterol y de triglicéridos, así como una disminución del colesterol unido a lipoproteínas de alta densidad (C-HDL), en más del 50\% de los pacientes estudiados, alcanzando hasta el $81.81 \%$ de alteración en las cifras de HDL en la esteatosis hepática ${ }^{8,9}$.

El síndrome metabólico se define por la presencia de al menos tres de los siguientes componentes $^{10,11}$ :

- Obesidad central: contorno de la cintura > $102 \mathrm{~cm}$ en hombres y $>88 \mathrm{~cm}$ en mujeres.

- Concentraciones elevadas de triglicéridos: $\geq 150 \mathrm{mg} / \mathrm{dl}$.

- Valores de C-HDL $\leq 40 \mathrm{mg} / \mathrm{dl}$ en hombres $\mathrm{y} \leq 50 \mathrm{mg} / \mathrm{dl}$ en mujeres.

- Presión arterial elevada: $\geq 130 / 85 \mathrm{mmHg}$.

- Glucosa en ayuno elevada: $\geq 100 / 110$ mg/dl.

Tomando en cuenta los criterios de diagnóstico de síndrome metabólico, existe la necesidad de que en los reportes de radiología se documenten adecuadamente los hallazgos en la densidad hepática y su relación con el riesgo de síndrome metabólico.
El objetivo de este estudio fue correlacionar los valores de $\mathrm{UH}$ reportados en el hígado con las alteraciones bioquímicas en pacientes asintomáticos.

\section{MÉTODO}

Estudio longitudinal y retrospectivo en pacientes que acudieron a estudios de imagenología en el Hospital Christus Muguerza de Alta Especialidad a quienes se realizó tomografía de abdomen y estudios de laboratorios de química clínica sanguínea en el periodo de enero de 2018 a agosto de 2019.

Se utilizaron un tomógrafo General Electric Revolution de 256 detectores (GE Milwaukee) y una estación de reformateo en el sistema RIS-PACS Carestream. Se colocaron regiones de interés (ROI) circulares de $1 \mathrm{~cm}$ de diámetro (Fig. 1) y se realizó un promedio total, el cual se integró a la base de datos. La medición del perímetro abdominal se obtuvo a nivel de L3 en un corte axial estricto (Fig. 2).

Los criterios de inclusión fueron:

- Pacientes que acudieron al Departamento de Radiología e Imagen del Hospital Christus Muguerza de Alta Especialidad de la ciudad de Monterrey con solicitud de tomografía de abdomen y que contaran con estudio de química sanguínea por el laboratorio de esta institución.

- Pacientes mayores de 18 años de edad, sin distinción de sexo.

- Pacientes sin distinción de índice de masa corporal.

- Pacientes sin tratamiento para EHGNA. 


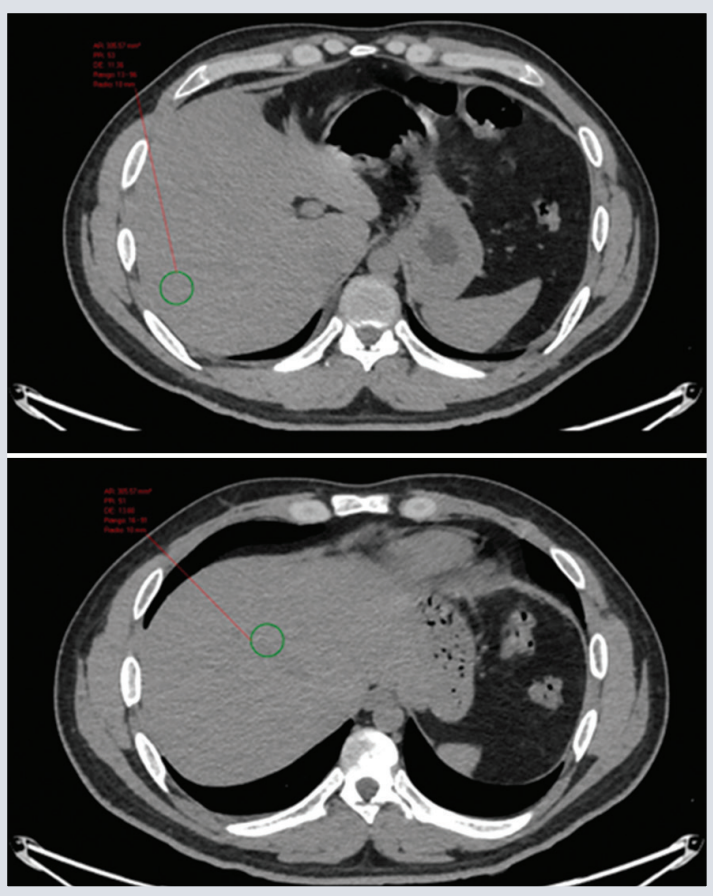

Figura 1. Ejemplo de ROI (región de interés) de $1 \mathrm{~cm}$ de diámetro colocado en los segmentos VI y VII.

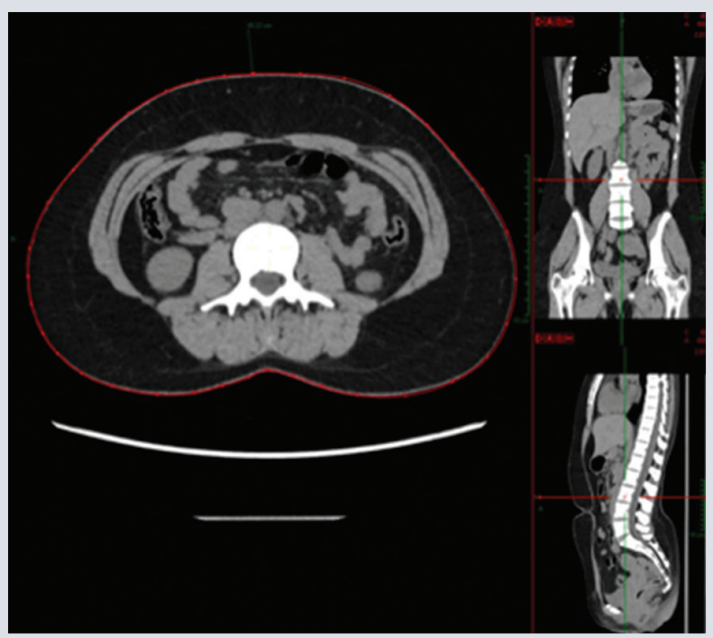

Figura 2. Medición del perímetro abdominal se obtiene a nivel de L3 en un corte axial estricto.

Los criterios de exclusión fueron:

- Pacientes con tratamiento de síndrome metabólico.
- Pacientes bajo tratamiento de EHGNA.

- Pacientes con antecedentes de alcoholismo.

- Pacientes con procedimientos invasivos en el hígado o las vías biliares.

Los datos recabados se analizaron mediante pruebas de correlacion de Pearson y tablas de contingencia y ROC (receiver operating characteristic).

Se clasificó el estudio según el Reglamento de la Ley General de Salud en Materia de Investigación para la Salud, Artículo N. 17, como sin riesgo por ser retrospectivo.

\section{RESULTADOS}

Se evaluaron 85 pacientes que cumplían con los criterios de ingreso, de los cuales ocho se excluyeron por no disponer de expediente completo, 12 por presentar patología quirúrgica y dos por tener variantes anatómicas que imposibilitan la adecuada colocación de las ROI para la medición de la totalidad de las UH. Finalmente, se incluyeron 63 pacientes (Tabla 1).

Se encontró una correlación estadísticamente significativa con la prueba $\mathrm{R}$ de Pearson entre los valores de UH, las alteraciones de las transaminasas y la presencia de síndrome metabólico (Tabla 2). Esta correlación es inversamente proporcional a los valores de UH y la elevación de las enzimas hepáticas y la presencia de síndrome metabólico.

Se realizó una curva ROC utilizando como parámetros los valores de UH promedio y la 
Tabla 1. Estadísticos descriptivos

\begin{tabular}{|l|c|c|c|}
\hline & Media & Desviación estándar & $\mathbf{n}$ \\
\hline UH promedio & 44.45 & 16.359 & 63 \\
\hline GGT & 73.49 & 195.862 & 63 \\
\hline $\begin{array}{l}\text { Síndrome } \\
\text { metabólico }\end{array}$ & 0.27 & 0.447 & 63 \\
\hline AST & 27.32 & 22.449 & 63 \\
\hline ALT & 30.54 & 25.216 & 63 \\
\hline Glucosa & 109.35 & 21.649 & 63 \\
\hline
\end{tabular}

ALT: alanina aminotransferasa; AST: aspartato aminotransferasa; GGT: gamma-glutamil transferasa.

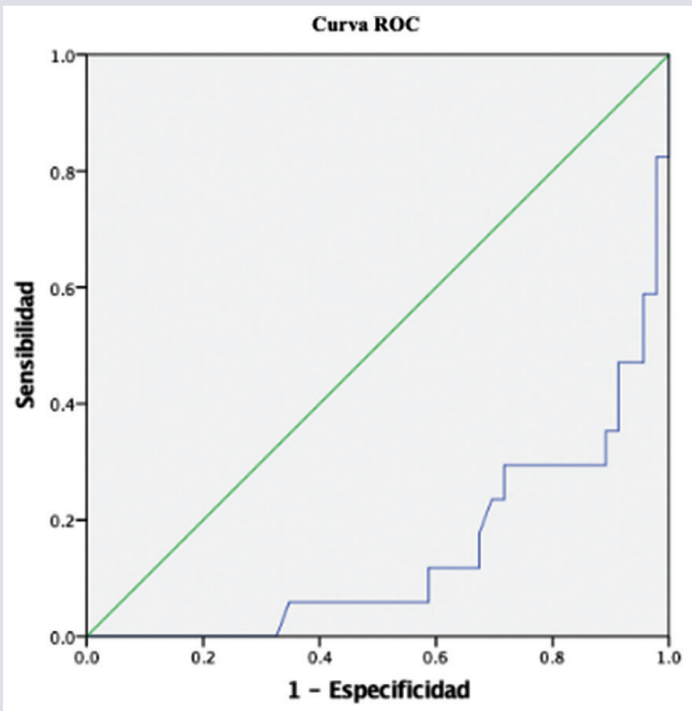

Los segmentos de diagonal se generan mediante empates.

Figura 3. Curva ROC para síndrome metabólico y unidades Hounsfield. $A U R O C=0.145$ con límite inferior de 0.041 y límite superior de 0.248. AUROC: area under the ROC curve.

presencia o no de síndrome metabólico (Fig. 3).

Al analizar las coordenadas de cruce de las UH y la presencia o no de síndrome metabólico, se encontró que el valor de 45.4 tiene mayor utilidad. En la muestra, con este punto de corte, se encontró una sensibilidad del 82\% y una especificidad del 72\% (Tabla 3).

\section{DISCUSIÓN}

En un estudio que incluyó 154 candidatos a trasplante de hígado, un valor CT-ls umbral de -9 tuvo una especificidad del 100\% y una sensibilidad del $82 \%$ para la detección de esteatosis hepática de moderada a grave ${ }^{5}$. Otro estudio informó que un umbral CT-1s de 3.2 tenía una sensibilidad del 72.7\% y una especificidad del $91.3 \%$. Al no existir un estadiaje por tomografía computada y su relación con el síndrome metabólico, se busca encontrar el promedio de $\mathrm{UH}$ en pacientes que cumplan los criterios de síndrome metabólico.

Se logró cuantificar el perímetro abdominal y el promedio de UH gracias al sistema de medición integrado en el RIS-PACS, colocando región de interés (ROI) circular de un centímetro de diámetro al centro de cada segmento hepático según la clasificación de Couinaud obteniendo así las mediciones promedio de Unidades Hounsfield de ese segmento de parénquima, posteriormente obteniendo un promedio de todos los segmentos.

De acuerdo con los resultados obtenidos, se encontró que sí existe una correlación entre el riesgo metabólico y el promedio de UH, pero sin lograr establecer un diagnóstico debido a su baja sensibilidad. Se logró identificar que existe una correlación inversamente proporcional de los valores de HU y las enzimas hepáticas. De acuerdo con la literatura, el médico radiólogo debe reportar como esteatosis hepática a pacientes con menos de $55 \mathrm{UH}$, pero al establecer el punto de corte en 
Tabla 2. Correlaciones de Pearson

\begin{tabular}{|l|c|c|c|c|c|c|} 
& UH promedio & GGT & Síndrome metabólico & AST & ALT & Glucosa \\
\hline UH promedio & & & & \\
Correlación de Pearson & 1 & $-0.358^{*}$ & $-0.604^{*}$ & -0.245 & $-0.454^{*}$ & -0.132 \\
Sig. (bilateral) & & 0.004 & 0.000 & 0.053 & 0.000 & 0.302 \\
n & 63 & 63 & 63 & 63 & 63 \\
\hline
\end{tabular}

ALT: alanina aminotransferasa; AST: aspartato aminotransferasa; GGT: gamma-glutamil transferasa.

*La correlación es significativa en el nivel 0.01 (bilateral).

Tabla 3. Sensibilidad y especificidad con el punto de corte de $45.5 \mathrm{UH}$

\begin{tabular}{|l|l|c|c|}
\hline & \multicolumn{2}{|c|}{ Corte 45.4 UH } & Total \\
\hline Estado de salud & \multicolumn{1}{|c|}{ Positivo } & Negativo & \\
\hline Sano & 13 & 33 & 46 \\
\hline Enfermo & 14 & 3 & 17 \\
\hline Sensibilidad & P+ (enfermo) & $82 \%$ & \\
\hline Especificidad & P- (sano) & $72 \%$ & \\
\hline Falso - & P- (enfermo) & $18 \%$ & \\
\hline Falso + & P+ (sano) & $28 \%$ & \\
\hline VP+ & VP / FP + VP & $52 \%$ & \\
\hline VP- & VN / FN + VN & $92 \%$ & \\
\hline
\end{tabular}

FN: falso negativo; FP: falso positivo; VN: verdadero negativo; VP: valor positivo.

45.4 UH, según los hallazgos de laboratorio en nuestra población, parece que no existe un riesgo significativo en los pacientes cuya densidad hepática es mayor que este punto de corte.

Se encontró que la mayor utilidad de la prueba es su valor positivo negativo, debido que para tener mayor sensibilidad, la prueba no logra discriminar entre individuos enfermos y los falsos positivos por sí sola.

De acuerdo con los resultados obtenidos, se sugiere aumentar el tamaño de muestra con el fin de establecer un mejor valor de corte para ayudar al radiólogo a hacer una mejor interpretación al reportar estudios, y ayudar al médico tratante a abordar de una mejor manera a su paciente.

\section{CONCLUSIONES}

De acuerdo con lo obtenido en este estudio, el radiólogo puede ayudarse para establecer como corte inferior el valor de $45.4 \mathrm{UH}$ en pacientes que cuentan con una alta probabilidad de no presentar alteraciones metabólicas, y de esta manera determinar y recalcar en el reporte radiológico cuando un paciente presente menor densidad, sugiriendo así un mayor enfoque en la evaluación clínica y de laboratorios por parte del médico tratante para descartar un síndrome metabólico.

\section{AGRADECIMIENTOS}

A todo el departamento de radiología e imagen del Hospital Christus Muguerza Alta Especialidad, por brindar un excelente grupo de trabajo.

\section{FINANCIAMIENTO}

No hubo financiamiento alguno para el artículo. 


\section{CONFLICTO DE INTERESES}

Los autores declaran no tener conflicto de intereses.

\section{RESPONSABILIDADES ÉTICAS}

Protección de personas y animales. Los autores declaran que para esta investigación no se han realizado experimentos en seres humanos ni en animales.

Confidencialidad de los datos. Los autores declaran que han seguido los protocolos de su centro de trabajo sobre la publicación de datos de pacientes.

\section{Derecho a la privacidad y consentimiento in-} formado. Los autores han obtenido el consentimiento informado de los pacientes y/o sujetos referidos en el artículo. Este documento obra en poder del autor de correspondencia.

\section{BIBLIOGRAFÍA}

1. Sung KC, Wild SH, Byrne CD. Development of new fatty liver, or resolution of existing fatty liver, over five years of follow-up, and risk of incident hypertension. J Hepatol. 2014;60:1040-5.

2. McCullough AJ. Non-alcoholic liver disease: natural history. En: Leushner U, James OFW, Dancygier H, editores. Steatohepatitis (NASH and ASH). Netherlands: Kluwer Academic; 2001. p. 11-20.

3. Castro Martínez MG, Banderas Lares DZ, Ramírez Martínez JC, Escobedode la Peña J. Prevalencia de hígado graso no alcohólico en individuos con síndrome metabólico. Cir Cir. 2012;80:128-33.

4. Kramer H, Pickhardt PJ, Kliewer MA, Hernando D, Chen GH, Zagzebski JA, et al. Accuracy of liver fat quantification with advanced CT, MRI, and ultrasound techniques: prospective comparison with MR spectroscopy. AJR Am J Roentgenol. 2017;208:92-110.

5. Lee SS, Park SH, Kim HJ, Kim SY, Kim MY, Kim DY, et al. Non-invasive assessment of hepatic steatosis: prospective comparison of the accuracy of imaging examinations. J Hepatol 2010;52:579-85.

6. Hindman N, Lee J, Babb JS. Multi-detector row CT attenuation measurements: assessment of intra and interscanner variability with an anthropomorphic body CT phantom. Radiology. 2007;242:109-19

7. Lee SS, Park SH. Radiologic evaluation of nonalcoholic fatty liver disease. World J Gastroenterol. 2014;20:7392-402.

8. Powell EE, Cooksley WG, Hanson R, Searle J, Halliday JW, Powell LW. The natural history of nonalcoholic steatohepatitis: a follow-up study of fortytwo patients for up to 21 years. Hepatology. 1990;11:74-80.

9. Xia MF, Yan HM, He WY, Li XM, Li CL, Yao XZ, et al. Standardized ultrasound hepatic/renal ratio and hepatic attenuation rate to quantify liver fat content: an improvement method. Obesity (Silver Spring). 2012;20:444-52.

10. Durán P, Piazza N, Trifone L, coordinadores. Consenso sobre factores de riesgo de enfermedad cardiovascular en pediatría. Obesidad. Arch Argent Pediatr. 2005;103:262-81

11. Lerman Garber I, Aguilar-Salinas CA, Gómez-Pérez FJ, Reza Albarrán A Hernández Jiménez S, Vázquez Chávez C, et al. El síndrome metabólico. Posición de la Sociedad Mexicana de Nutrición y Endocrinología sobre la definición, fisiopatología y diagnóstico. Características del síndrome metabólico en México. Revista de Endocrinología y Nutrición. 2004;12:109-22. 